\title{
Infrared spectral sequence of quenched carbonaceous composite subjected to thermal annealing
}

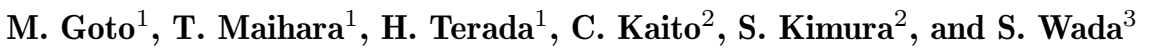 \\ 1 Department of Physics, Kyoto University, Kitashirakawa Oiwake-cho, Sakyo, Kyoto 606-8502, Japan \\ e-mail: mgoto@cr.scphys.kyoto-u.ac.jp \\ 2 Department of Physics, University of Ritsumeikan, Nojicho, Kusatsu, Shiga 525-8577, Japan \\ 3 Department of Chemistry, University of Electro-Communications, Chofugaoka, Chofu-shi, Tokyo 182-8585, Japan
}

Received August 30; accepted October 4, 1999

\begin{abstract}
We investigate the spectroscopic alteration through thermal annealing on the quenched carbonaceous composite (QCC) produced from hydrocarbon plasma. The emission and absorption spectra of QCC samples annealed at various temperatures indicate that the proportion of aliphatic $\mathrm{C}-\mathrm{H}$ bonds in $\mathrm{QCC}$ decreases relative to aromatic $\mathrm{C}-\mathrm{H}$ bonds with increase of annealing temperature. By comparing the spectra of annealed QCC with observed emission spectra of proto-planetary nebulae, similarity between the spectral sequence obtained in the laboratory and that in the observational data is clearly noticed. On this basis, we discuss a simple evolutional scenario of carbon dust in circumstellar environment around cool evolved stars.
\end{abstract}

Key words: (stars:) circumstellar matter — stars: individual (IRAS 05341+0852) — stars: AGB and postAGB - (ISM:) dust, extinction — infrared: ISM: lines and bands — methods: laboratory

\section{Introduction}

The unidentified infrared bands (UIR bands) considered ubiquitous where the interstellar carbonaceous dusts are exposed to UV radiation fields have a variety of spectroscopic appearances. Based on the $3 \mu \mathrm{m}$ spectra of various sources, Geballe (1997) and Tokunaga (1997) proposed a classification of UIR sources made of several groups. The class $\mathrm{A}$ is the most common type of spectra characterized by intense emission at $3.3 \mu \mathrm{m}$ with weaker subsidiary peaks seen in the $3.4-3.6 \mu \mathrm{m}$. The class B and class $\mathrm{C}$ have relatively stronger emission in the $3.4-3.6 \mu \mathrm{m}$,

Send offprint requests to: M. Goto

Correspondence to: mgoto@cr.scphys.kyoto-u.ac.jp showing a broad $3.4 \mu \mathrm{m}$ feature which defined as class B, and sharp emission peaks at $3.43 \mu \mathrm{m}$ and $3.53 \mu \mathrm{m}$ defined as class $\mathrm{C}$.

All the class B sources are carbon rich proto-planetary nebulae (PPNe). A PPN is an evolved transitional object that ceased violent mass loss in the AGB phase, but the central star has not become hot enough to ionize the circumstellar material to form a planetary nebula (Kwok 1993). The class B sources are certainly a minor population of the UIR objects. However, we consider the class B sources as the key to understand the open questions on the creation and evolution of UIR carrier because a PPN is one of the main sites of dust formation. It is also interesting to note that there is a sample of PPNe that has isolated $3.3 \mu \mathrm{m}$ band without $3.4 \mu \mathrm{m}$ emission as The Red Rectangle (HD 44179) (Tokunaga et al. 1991). There is also large scattering in the intensity of $3.4 \mu \mathrm{m}$ band relative to $3.3 \mu \mathrm{m}$ band in those PPNe classified in class B.

We infer the spectral diversity of PPNe is the link to connect class B spectral features with class A. We searched for a laboratory analog of carbon material which has spectral flexibility required to reproduce the variation of PPNe spectra. Sakata et al. (1990), Dischler et al. (1983), and Bounouh et al. (1995) demonstrated that the intensity of the $3.4 \mu \mathrm{m}$ absorption band decreases relative to the $3.3 \mu \mathrm{m}$ band in amorphous carbon material by heating. Scott \& Duley (1996), and Scott et al. (1997) also reported the similar thermal alteration in the absorption and emission features of hydrogenated amorphous carbon (HAC) prepared by laser ablation of graphite. We produced QCC films with a plasma vapor deposition technique developed by Sakata et al. (1984) which intends to mimic the carbon dust solidification in expanding carbon rich stellar atmosphere. In this paper, we investigated the effect of thermal annealing on QCC in terms of emission and absorption spectra. We compare the spectral sequence of thermally 

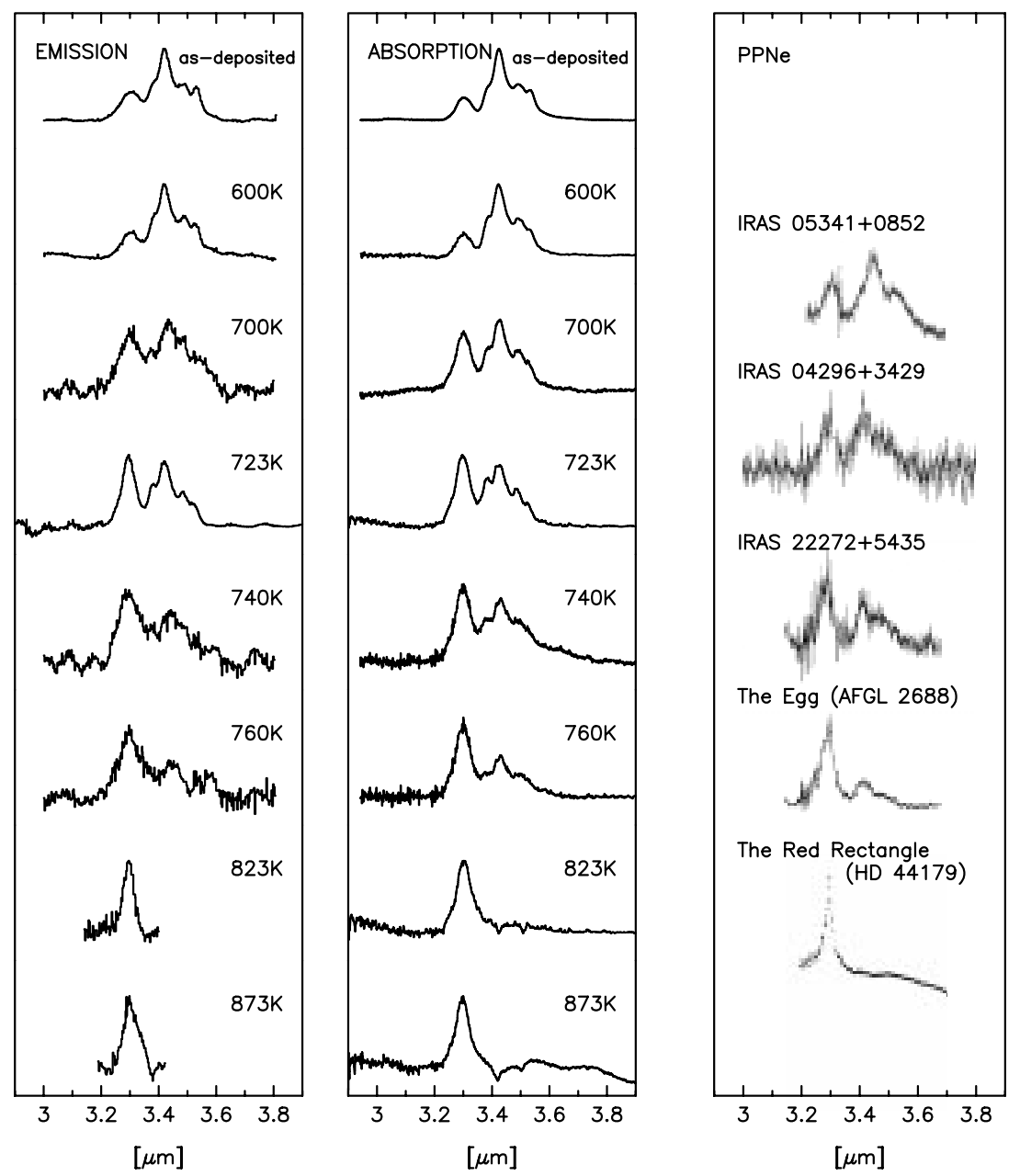

Fig. 1. The spectral sequence of QCC along with temperatures of thermal annealing. The emission and absorption spectra are shown in the left and the middle panels, respectively, in the order of annealing temperature; as-deposited, 600, 700, 723, 740, 760,823 , and $873 \mathrm{~K}$ from the top to the bottom. The spectra of several PPNe are shown in the right panel for comparison adapted from Joblin et al. (1996), Geballe et al. (1992), and Tokunaga et al. (1991)

annealed QCC with the spectral variation of PPNe in the $3 \mu \mathrm{m}$ region to discuss the circumstellar evolution of carbon dust.

\section{Experiments}

The production method of QCC described in detail by Sakata et al. (1984) is summarized here. The QCC condensate is prepared by the technique of hydrocarbon plasma deposition. The source gas of methane is ionized by microwave discharge, and is spouted out into a vacuum chamber through a small nozzle. The plasma gas adiabatically expands and is immediately quenched to solid material. The condensate is deposited onto $\mathrm{NaCl}$ or $\mathrm{CaF}_{2}$ substrates placed in the vacuum chamber. The $\mathrm{NaCl} / \mathrm{CaF}_{2}$ substrates are used because of high transmittance and no spectral features in the $3 \mu \mathrm{m}$ region.

Then the QCC films are moved into an evacuated quartz tube, and set in an electronic furnace. The tem- perature of the furnace is raised at a rate of $5 \mathrm{~K}$ per minute to a predetermined temperature. The sample is heated in the furnace at the temperature for 20 minutes. A portion of deposited QCC film evaporates by heating, and re-sticks to the cooler part of the quartz tube. The amount of evaporation grows with the annealing temperature, for instance, more than $90 \%$ in mass is lost from the fresh deposition after the annealing at $800 \mathrm{~K}$. We adjust the duration of deposition process five to ten times longer than the sample for no annealing in order to prepare the thicker deposition for annealing at higher temperature. As a result we obtain eight samples at the temperatures of $600,700,723,740,760,823,873 \mathrm{~K}$ as well as as-deposited film whose absorptions are fairly constant $(\sim 15 \%)$.

In order to obtain the thermal emission spectra of QCC we use a near-infrared spectrograph originally developed for astronomical observations Imanishi et al. (1996). The spectrograph is equipped with an InSb detector array and covers $2.8-4.2 \mu \mathrm{m}$ in a single exposure with a wavelength 
resolution of about 1300 . The annealed and as-deposited samples are fixed in a holder in front of the spectrograph window, and heated by a ceramic resistance that contacts to the substrate. So as to reduce the background emission from the environment, a liquid nitrogen surface is viewed through a mirror put behind the sample holder. We measure the temperature of the emitting material with Pt resistance attached to the substrate.

A net spectrogram of QCC sample is obtained by subtracting a spectrum of a blank substrate. The sensitivity of the spectrograph is calibrated by measuring thermal emission spectrum of the room temperature assuming a blackbody emission. The baseline of the spectrum is fitted with spline function and subtracted to compare the extracted spectral features with the observational data.

The absorbance of QCC films is taken by a Shimazu FTIR - 8600 PC spectrophotometer at room temperature with a wavelength resolution of about 1500 . The baseline of the absorption spectra attributable to the the net continuum absorption of QCC and substrates are fitted with spline function and subtracted.

\section{Results}

We show the spectral sequence of the thermally annealed QCC in the $3 \mu \mathrm{m}$ region in Fig. 1. The emission and absorption spectra are collected in the left and the middle panels respectively. The spectra are arranged in order of the annealing temperature from the top to the bottom. In the right panel, we display spectra of PPNe with various spectroscopic characters for comparison.

In Fig. 2 we decompose the emission spectrum of asdeposited film into five Gaussian components of various $\mathrm{C}-\mathrm{H}$ vibrational modes according to the band assignment in the literatures on amorphous carbon (Dischler et al. 1983; Bounouh et al. 1995). The band centered at $3.30 \mu \mathrm{m}$ is assigned to the aromatic $\mathrm{sp}^{2} \mathrm{CH}$ stretching mode blended with the olefinic $\mathrm{sp}^{2} \mathrm{CH}_{2}$. The bands at $3.38,3.42,3.48 \mu \mathrm{m}$ are due to symmetric and antisymmetric stretching $\mathrm{C}-\mathrm{H}$ vibrations in $\mathrm{sp}^{3} \mathrm{CH}_{2}$ and $\mathrm{CH}_{3}$ chemical groups. The assignment of the $3.53 \mu \mathrm{m}$ band is somewhat uncertain.

In Fig. 3 we present the overall thermal emission of QCC samples before and after the thermal annealing at $740 \mathrm{~K}$. The $\mathrm{CaF}_{2}$ substrates used in the measurement have very low emissivity and no spectral features in the wavelength region, and furthermore we cancel out the minor contribution due to the substrate by subtracting its spectrogram from that of the QCC sample. Thus we consider the emissions observed here are certainly originated from QCC material itself. The absolute intensity of the QCC emission is calibrated by blackbody radiation of room temperature measured in the same configuration. Note that the QCC sample before and after the annealing is not equal in quantity. The emission features at $3.3-3.6 \mu \mathrm{m}$ are by far strong in the as-deposited QCC.

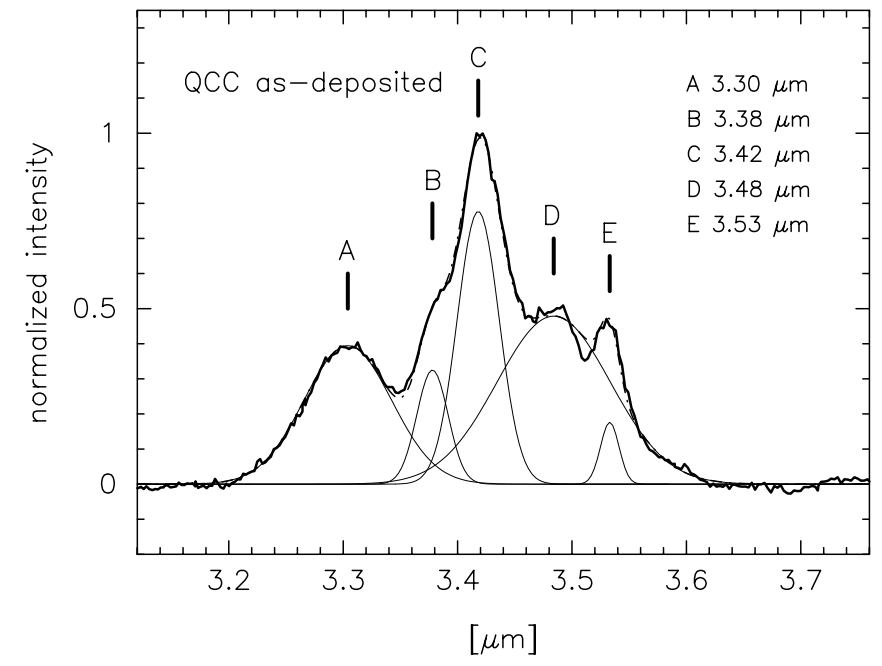

Fig. 2. The emission spectrum of an as-deposited QCC sample decomposed into five Gaussian components. The band centered at $3.30 \mu \mathrm{m}$ is assigned to the aromatic $\mathrm{sp}^{2} \mathrm{CH}$ stretching mode blended with the olefinic $\mathrm{sp}^{2} \mathrm{CH}_{2}$. The bands at $3.38,3.42$, $3.48 \mu \mathrm{m}$ are due to symmetric and antisymmetric stretching $\mathrm{C}-\mathrm{H}$ vibrations in $\mathrm{sp}^{3} \mathrm{CH}_{2}$ and $\mathrm{CH}_{3}$ chemical groups. The assignment of the $3.53 \mu \mathrm{m}$ band is somewhat uncertain. The sum of the Gaussian components is shown in dot-dashed line

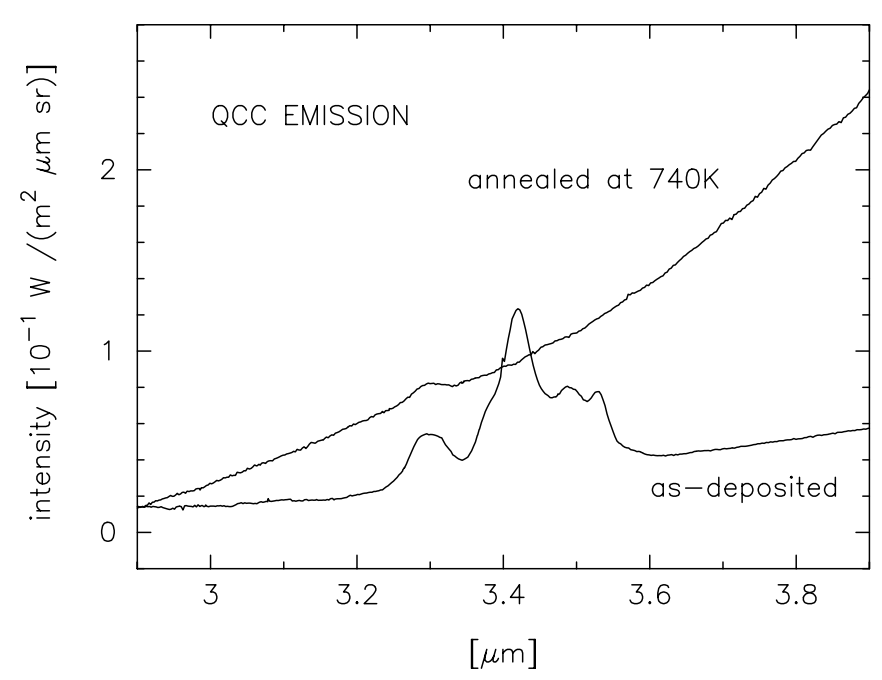

Fig. 3. The over all emission spectra of QCC samples including continuum emission

\section{Discussion}

\subsection{Emission spectra of $Q C C$}

This paper is the first report of infrared emission spectra of QCC. Since the UIR bands are mostly observed in emission, it is particularly important for the proposed laboratory material of UIR bands carrier to positively demonstrate whether it does emit when exposed in an environment of thermal or radiative excitations.

In Fig. 4 we compare emission and absorption spectra of QCC annealed at $723 \mathrm{~K}$. Both spectra are almost 


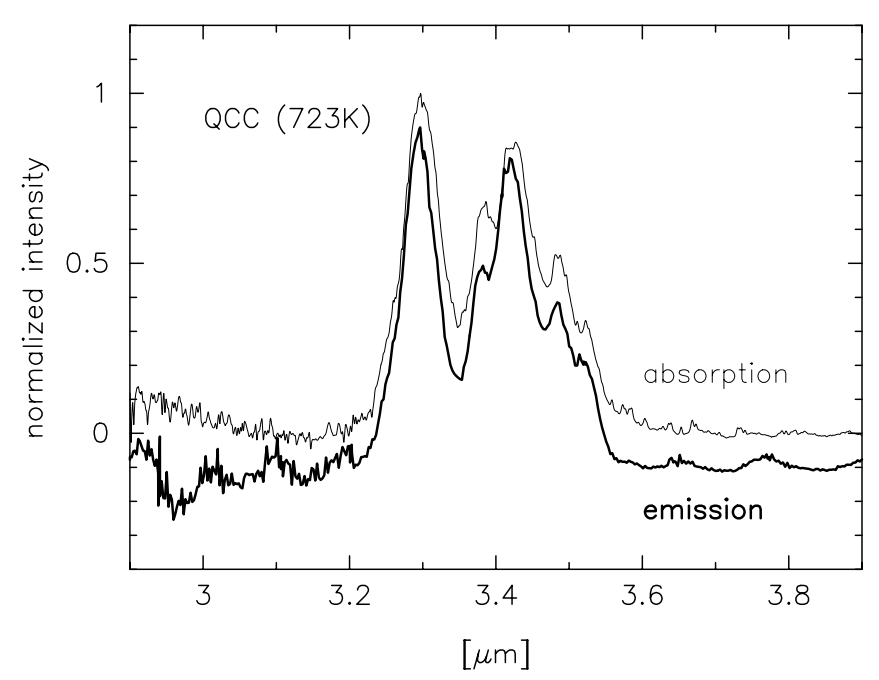

Fig. 4. The emission (the bold line) and absorption (the thin line) spectra of QCC annealed at $723 \mathrm{~K}$. The spectra are displaced in ordinate for clarity

identical in the respective profiles. The coincidence suggests that emissions are basically the transition from upper vibrationally excited states to ground states in various modes; not being associated with so-called hot bands. We conclude that the broad and intense emission band at the $3.4-3.6 \mu \mathrm{m}$ region toward class B sources is surely not due to a hot band or combined effect of them.

The measured temperatures of the samples in emission are between $310 \mathrm{~K}$ and $370 \mathrm{~K}$ in the present experiment. On the other hand the temperature at the inner edge of the dust shell under a typical PPN condition is calculated as,

$$
\begin{aligned}
T_{\mathrm{d}} & =\left(\frac{L}{16 \pi \sigma r_{\mathrm{d}}^{2}}\right)^{1 / 4} \\
& \sim 1.110^{2} \cdot\left(\frac{L / 10^{4} L_{\odot}}{\left(r_{\mathrm{d}} / 10^{16} \mathrm{~cm}\right)^{2}}\right)^{1 / 4}
\end{aligned}
$$

where $L, \sigma$, and $r_{\mathrm{d}}$ represent the luminosity of the central star, Stephan-Boltzmann constant, and the distance from the central star to the dust grain respectively. Assuming typical physical parameter for a surrounding dust shell of a PPN, the temperature of the dust grain embedded in the shell is approximately $100 \mathrm{~K}$. Several authors have derived even higher temperature of the dust shells around PPNe to be $150-250 \mathrm{~K}$ based on spectroscopic and imaging observations with more realistic theoretical models (Volk \& Kwok 1989; Meixner et al. 1997; Szczerba et al. 1997). Therefore we consider it is not unrealistic for the dust grains in PPN shells to emanate UIR emission band in thermally equilibrium state with the radiation from the central star rather than by stochastic heating with a single UV photon.

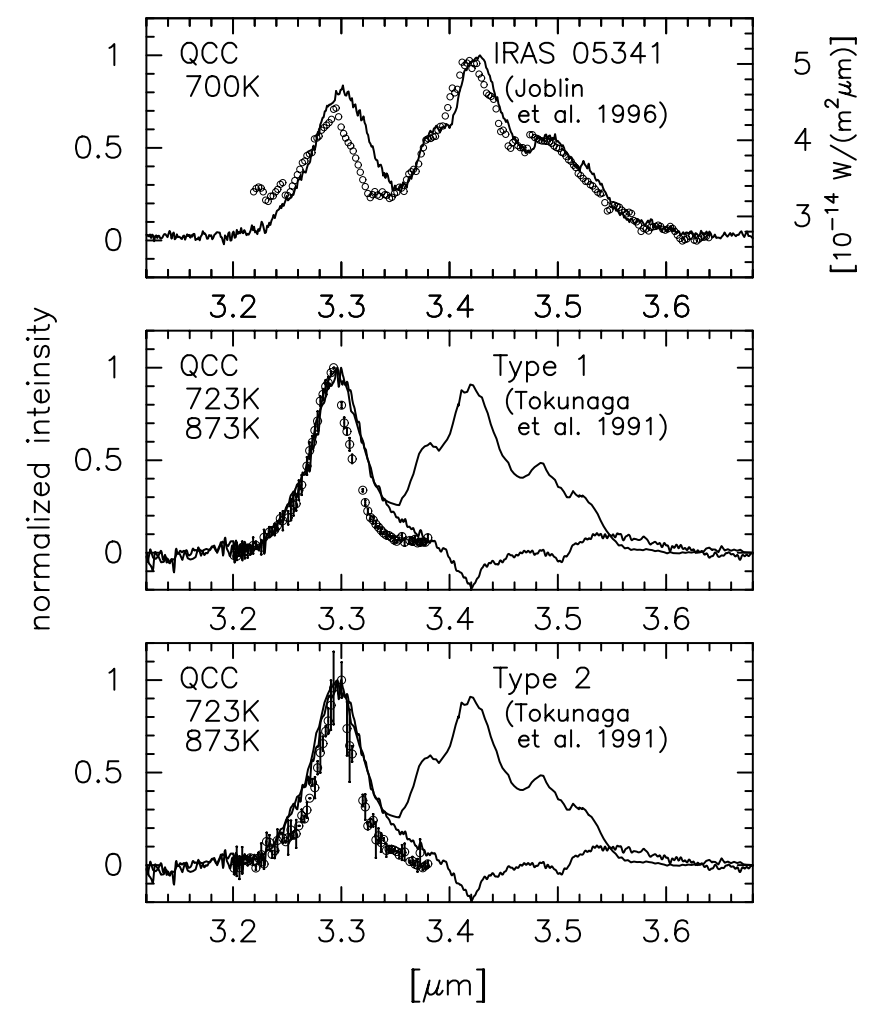

Fig. 5. The comparison of the spectral profiles between observations (the open circles) and the annealed QCC samples (the solid lines) at $700 \mathrm{~K}$ for IRAS $05341+0852$ (Joblin et al. 1996), at $723 \mathrm{~K}$ and $700 \mathrm{~K}$ for synthetics $3.3 \mu \mathrm{m}$ band profiles of Type 1 and Type 2 (Tokunaga et al. 1991)

\subsection{Spectral profiles of $Q C C$}

The most characteristic feature of the infrared spectra of QCC as a laboratory analog of carbon dust is the complex spectral profiles in the $3.4-3.6 \mu \mathrm{m}$ region, especially seen in the spectra of as-deposited QCC sample. In Fig. 5 we examine the match of the spectral profiles between observational spectra and those of annealed QCC in detail. The absorption spectra of QCC are used for comparison except the emission spectra of the sample annealed at $723 \mathrm{~K}$ because of higher signal to noise ratio than emission spectra. We find the fairly good coincidence between the spectra of IRAS $05341+0852$ (Joblin et al. 1996) and that of the QCC annealed at $700 \mathrm{~K}$ particularly in the $3.4-3.6 \mu \mathrm{m}$ region. The QCC spectrum precisely reproduces not only the overall band intensity of $3.4 \mu \mathrm{m}$ region relative to the $3.3 \mu \mathrm{m}$ band but also the shoulder at $3.38 \mu \mathrm{m}$ as well as the weaker peak near the $3.48 \mu \mathrm{m}$. The intense and complex bands in the $3.4 \mu \mathrm{m}$ region are one of the spectral characteristics which can not be reproducible by polycyclic aromatic hydrocarbon (PAH) molecules by themselves.

We also compare the profiles of prototypic $3.3 \mu \mathrm{m}$ emission bands of Type 1 and 2 presented by Tokunaga et al. (1991) with the spectra of QCC annealed at $723 \mathrm{~K}$ and $873 \mathrm{~K}$. We can see the excellent match between the 


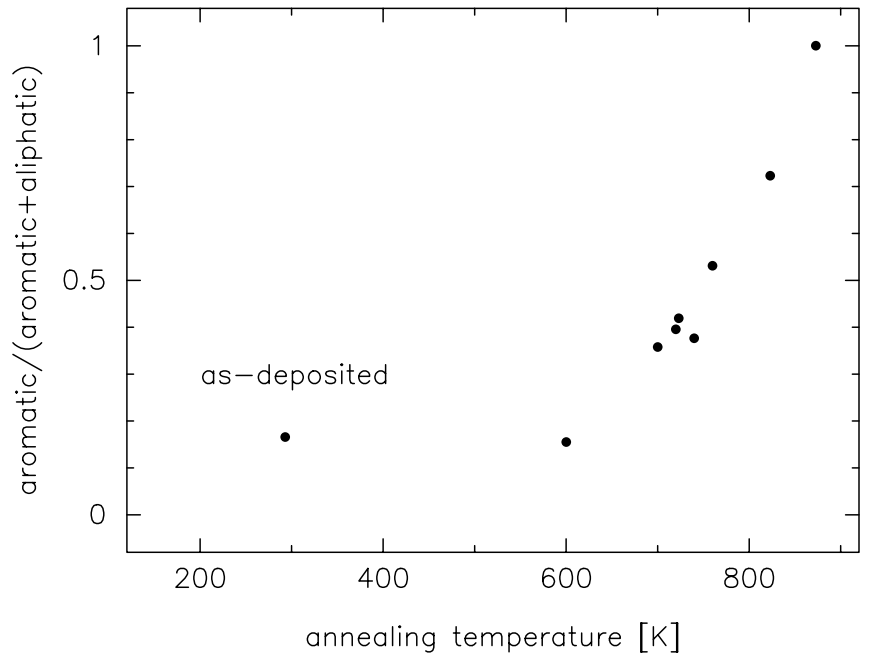

Fig. 6. The relative strength of aromatic $\mathrm{C}-\mathrm{H}$ bonds to the whole feature intensity. The 3.2 to $3.6 \mu \mathrm{m}$ spectra are fitted by five Gaussian components (Fig. 2) to obtain the individual band intensities

slopes at the shorter wavelength tails of the observed $3.3 \mu \mathrm{m}$ band and those of the QCC samples. In each case, the QCC spectrum exhibits significant excess above the observational data at the longer wavelength side of the $3.3 \mu \mathrm{m}$ band. Although the exact cause for the discrepancy is currently uncertain, it may be possibly due to the insufficient subtraction of the continuum spectra or a band broadening due to the interaction between chemical groups as a consequence of the measurement in a bulky film (Joblin et al. 1994).

\subsection{Spectral sequence of annealed $Q C C$}

Figure 6 illustrates the thermal transition of band intensity due to $\mathrm{sp}^{2}$ hybridized $\mathrm{CH}$ (aromatic $\mathrm{C}-\mathrm{H}$ ) bonds relative to the sum of $\mathrm{sp}^{2}$ and $\mathrm{sp}^{3}$ (aliphatic $\mathrm{C}-\mathrm{H}$ ) bonds calculated by integrating the decomposed bands intensity (Fig. 2). It might be incorrect to attribute whole intensity of $3.4-3.6 \mu \mathrm{m}$ emission to aliphatic $\mathrm{C}-\mathrm{H}$ bonds because the possibility of inclusion of small PAH molecules, whose aromatic $\mathrm{C}-\mathrm{H}$ bonds would contribute to $3.4-3.6 \mu \mathrm{m}$ features, cannot be excluded. However, aromatic $\mathrm{C}-\mathrm{H}$ features of the molecular species mostly appear at shorter wavelength than $3.4 \mu \mathrm{m}$, so we may safely divide the feature intensity into aliphatic $\mathrm{C}-\mathrm{H}$ and aromatic $\mathrm{C}-\mathrm{H}$ in terms of the band wavelength.

It is obvious that the band arise from aromatic $\mathrm{C}-\mathrm{H}$ bonds begins to increase relative to the whole feature intensity when heated up over $600 \mathrm{~K}$. The proportion of the band intensity continues to rise up to annealing at $\sim 900 \mathrm{~K}$ where the whole band strength is attributable to purely aromatic $\mathrm{C}-\mathrm{H}$ bonds. It must be borne in mind that the increase of the aromatic band ratio is the combined effect of two independent consequences of

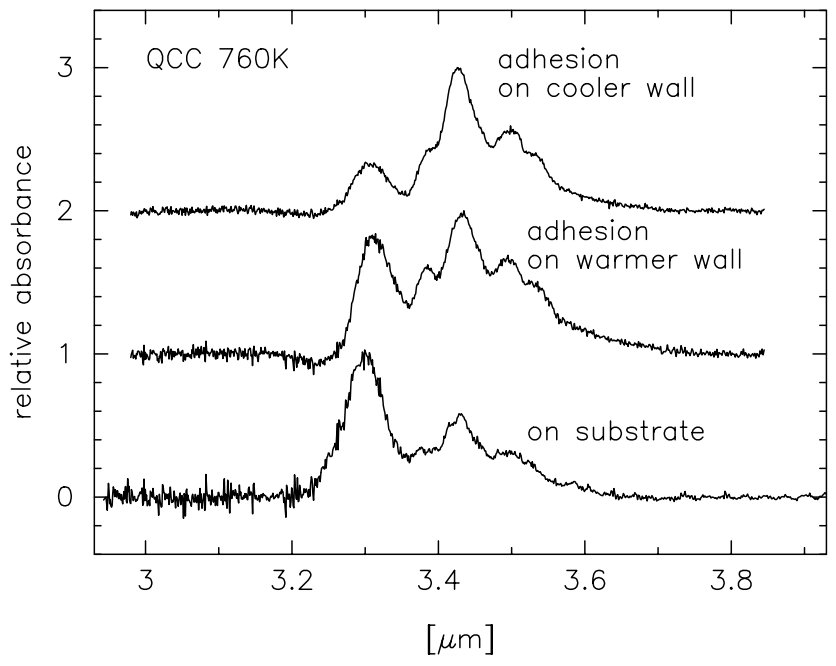

Fig. 7. The absorption spectrum of evaporated material when the QCC samples are heated in a evacuated quartz tube. The spectra indicate the material is rich with aliphatic $\mathrm{C}-\mathrm{H}$ bonds

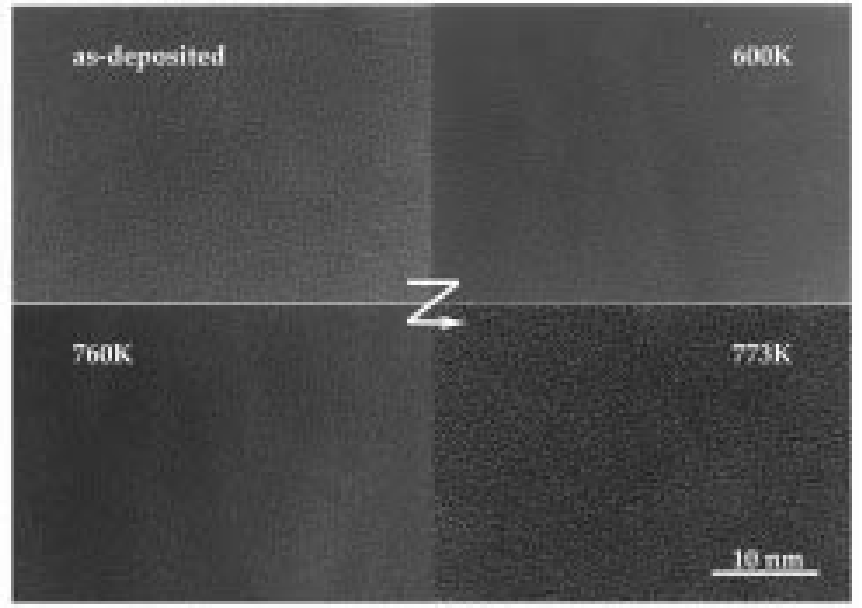

Fig. 8. HRTEM images of as-deposited and thermally annealed QCC. We can see curved stratified layers in the sample annealed at $760 \mathrm{~K}$, and carbon onion-like spherules at $823 \mathrm{~K}$. A scale bar of $10 \mathrm{~nm}$ is imposed in the panel

thermal annealing process. First it can be attributed to the chemical structural transformation of aliphatic carbon skeleton to aromatic or graphitic one. It is supported by Robertson (1991) and Dischler et al. (1983) who claim the graphitization of amorphous carbon material subjected to thermal annealing. In the meantime, when we heat the QCC sample in an evacuated quartz tube, comparatively volatile component of QCC evaporates from the film and re-adheres cooler wall of quartz tube. We collect the evaporated material, and examine the absorption spectra to find that the adhesion is rich with aliphatic $\mathrm{C}-\mathrm{H}$ bonds (Fig. 7). Therefore it is possible the effect shown in Fig. 6 would be explained not by the net increase of aromatic $\mathrm{C}-\mathrm{H}$ bonds but by the relative decrease of aliphatic $\mathrm{C}-\mathrm{H}$ bonds. Nevertheless we consider the formation of 
aromatic $\mathrm{C}-\mathrm{H}$ bonds occurs at least with significant contribution because we have confirmed the existence of newly formed carbon onion-like particles which consist of concentric spherical graphitic shells in the HRTEM micrograph of the annealed QCC sample. The HRTEM images of the as-deposited and annealed QCC are shown in Fig. 8 in the sequence of the annealing temperature. No periodic structure can be seen in the as-deposited film and the sample annealed at lower temperature. However, several stratified layers with curvature appear in the sample annealed at $760 \mathrm{~K}$. We can clearly see a number of carbon onion-like spherules in the sample annealed at $823 \mathrm{~K}$. We assume the growth of $\mathrm{sp}^{2}$ hybridized aromatic rings or formation of carbon onion-like spherules is the main cause for the spectroscopic transformation of QCC in the following discussion.

We compare the spectral sequence of the annealed QCC with the various spectra of PPNe in Fig. 1. We can see a remarkable similarity between the laboratory spectra and the observational data. The QCC spectra in each stage of thermal annealing, which gradually lose $3.4 \mu \mathrm{m}$ band, fairly closely trace the corresponding PPNe spectra. It is noteworthy that the variety of PPNe spectra can be systematically reproduced by the QCC samples by themselves; from IRAS $05341+0852$ with predominant $3.4 \mu \mathrm{m}$ emission to the Egg and the Red Rectangle with slight or no $3.4 \mu \mathrm{m}$ bands.

The thermal annealing process also affects the feature to continuum ratio significantly. The $3.2-3.6 \mu \mathrm{m}$ features are by far prominent in as-deposited QCC sample, in the same time continuum emission levels uprise after the annealing (Fig. 3). The strong continuum emission may be directly related with $\mathrm{sp}^{2}$ hybridized bond in the sample.

It is especially interesting to note that the UIR band intensity of PPNe relative to the continuum also drops off as the $3.4 \mu \mathrm{m}$ band intensity decreases relative to the $3.3 \mu \mathrm{m}$ band. The spectra of three extreme class B sources observed by Joblin et al. (1996) and Geballe et al. (1992) are shown in Fig. 9 in the sequence of $3.4 \mu \mathrm{m}$ band intensity relative to the $3.3 \mu \mathrm{m}$ band. The spectra are divided by blackbody continuum. It can be seen that the feature intensity normalized to the continuum diminishes in the sequence. We suppose the common behavior of the feature to continuum ratio strongly suggests the common nature between the carrier of the UIR band toward the class B sources and the thermally annealed QCC.

\subsection{Evolution of carbon dust}

The spectral similarity between the laboratory analog and the observational data leads us to a possible evolutionary scenario of carbon dust around PPNe, which has the same spectral effect in the $3 \mu \mathrm{m}$ region as the thermal annealing. We would like to interpret that the variation of PPN spectra should reflect the different stages of the evolution of carbon dust.

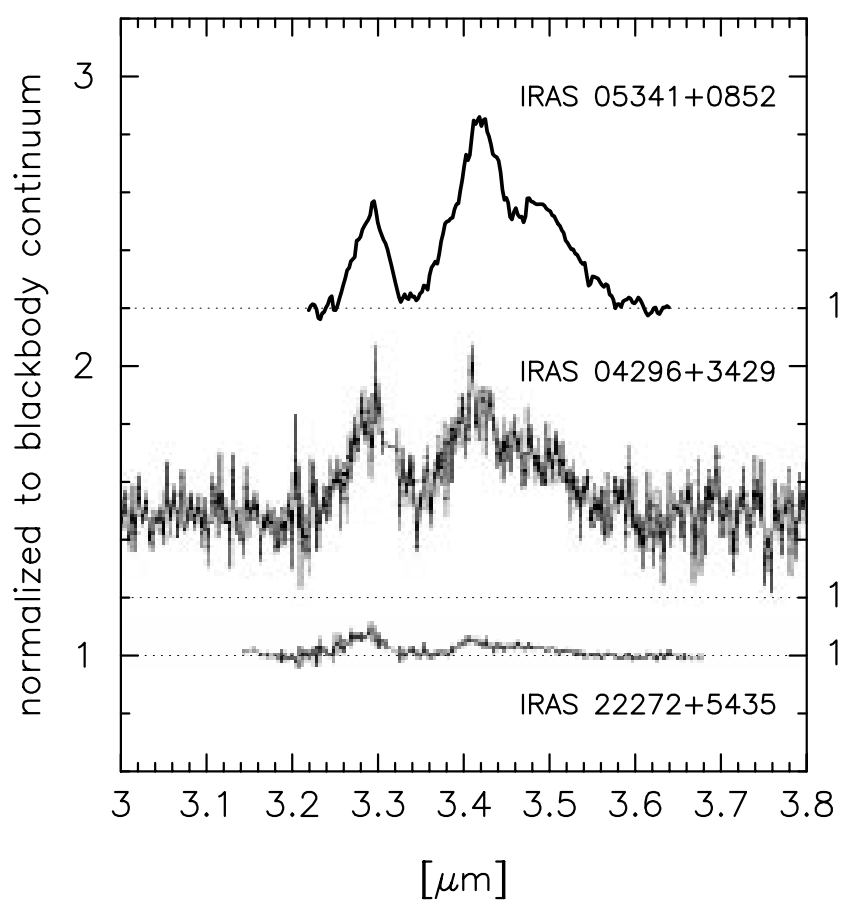

Fig. 9. The spectra of the extreme class B sources adopted referring to Joblin et al. (1996) and Geballe et al. (1992). They are obtained by being divided by underlying continuum which are assumed as blackbody. The continuum levels are indicated by dotted lines

The following is a proposed working hypothesis of carbon dust formation and evolution. (1) Carbon dust is solidified as QCC-like amorphous material with rich $\mathrm{sp}^{3}$ hybridized $\mathrm{CH}$ bonds in the vicinity of the evolved carbon star. The material shows complex $3.4 \mu \mathrm{m}$ emission which is similar to that of IRAS $05341+0852$ or a QCC film immediately after the deposition. (2) The solidified carbon dust is then suffered from annealing. The intensity of $3.4 \mu \mathrm{m}$ emission decreases because of transformation of aliphatic $\mathrm{C}-\mathrm{H}$ bonds to the aromatic one. The emission band at $3.4 \mu \mathrm{m}$ is diminished before the carbon dust leaves the star to diffuse interstellar medium. (3) The aliphatic $\mathrm{C}-\mathrm{H}$ bonds that survive through the destructive annealing would contribute $3.4-3.6 \mu \mathrm{m}$ plateau emission of typical class A UIR spectra observed toward HII regions, reflection nebulae, and planetary nebulae.

The first point of the scenario is that it describes a fresh carbon dust as an amorphous material which contains rich aliphatic $\mathrm{C}-\mathrm{H}$ bonds. The second point is that it refers the destruction of $\mathrm{sp}^{3}$ hybridized $\mathrm{CH}$ bonds which separately occurs from the dust formation.

Frenklach \& Feigelson (1989) and Cherchneff et al. (1992) have claimed that it requires exceptional physical and chemical conditions such as high density of seed $\mathrm{C}_{2} \mathrm{H}_{2}$ molecules and slow mass loss velocity to produce sufficient amount of PAH directly out of a stellar atmosphere. We infer the condensation and subsequent chemical reaction 
to aromatization may provide a round way to avoid the difficulty of direct formation of aromatic material.

The size distribution of the particles is also affected by the thermal annealing. By heating, carbon onion-like spherules of certain diameter are produced out of a QCC film which has no specific partitional scale immediately after the deposition. When a dust model is given, the size of the grains is one of a few parameters that are determinable in fairly stringent way from observations. The size distribution of dust grains is considered to be controlled by (1) nucleation and growth rate, and (2) grain destruction by collision and shattering. The latter is known to provide a secure ground of the power law size distribution required for standard comprehensive dust models (Mathis et al. 1977). On the other hand as concerns well-known $2175 \AA$ A bump in UV interstellar extinction curve, the constant band center independent of the line of sight together with the narrow bump profiles prefers graphite grains to be highly uniform in size and shape (Draine 1989). The fragmentation into carbon onion-like spherules from amorphous material might be a process disregarded so far which controls the shape and the size of dust grains.

The compositional change of carbon dust from circumstellar to interstellar environment is previously pointed out by Buss et al. (1993) who argued the systematic spectral variations through AGB, PPN, to planetary nebula in the mid infrared region. Duley (1995) proposed an evolutional model characterized by the formation of featureless amorphous carbon in AGB phase, subsequent photochemical rehydrogenation to HAC in the presence of UV radiation, and possible dissociation to PAH molecules in a planetary nebula shock. Schnaiter et al. (1999) gave a support to the idea by demonstrating the production of nano-sized carbon particles with laser pyrolysis of $\mathrm{C}_{2} \mathrm{H}_{2}$ that have only weak $\mathrm{C}-\mathrm{H}$ features. On the other hand a lot of carbon rich PPNe do have $3.3 \mu \mathrm{m}$ emission due to aromatic $\mathrm{C}-\mathrm{H}$ bonds and in several cases additional broad $3.4 \mu \mathrm{m}$ emission due to aliphatic $\mathrm{C}-\mathrm{H}$ bonds. The spectral types of the most of the central stars of PPNe are found to be $\mathrm{F}-\mathrm{G}$ by spectroscopic observations in visible (Hrivnak et al. 1995). It is not likely that those PPNe are able to provide sufficient UV photons required to promote the active hydrogenation of amorphous carbon. The lack of plausible hydrogenation mechanism of amorphous carbon would be alleviated by our result that reveals the carbonaceous material rich in aliphatic $\mathrm{C}-\mathrm{H}$ bonds can be directly produced by hydrocarbon plasma deposition. Chiar et al. (1998) proposed the condensation of aliphatic hydrocarbon around PPNe and postprocessing to aromatic dust based on the infrared spectroscopy of AFGL 618 which exhibits an absorption feature at $3.4 \mu \mathrm{m}$ together with the weak $3.3 \mu \mathrm{m}$ emission feature. The transformation of aliphatic hydrocarbon to aromatic material is exactly what is observed in our thermal annealing. Although Chiar et al. (1998) suggested the photochemical reaction driven by FUV radiation as the promoter of the conversion, we would like to imply that the alternative thermal process also enables the spectroscopic transition.

What is not explained in our scenario is the lack of hydrocarbon features toward carbon rich AGB stars. The dust emission observed in the far infrared region clearly ensures the existence of large amount of dust around those progenitors of PPNe. The previous evolutional models attribute the featureless continuum toward AGB stars to amorphous carbon grains with little $\mathrm{C}-\mathrm{H}$ bonds. We speculate if the dust formation mechanism is possibly not continuous between the AGB and PPN phase. A certain mode-switching synchronized with the end of the main mass loss phase could accompany with the changes in the physical and chemical circumstances.

The thermal evolution proposed above requires heating of dust up to $\sim 800 \mathrm{~K}$ after the solidification. Here we conclude by proposing three possibilities such heating could occur. That is, (1) stochastic heating of a dust grain impinged by a single energetic photon radiated either from a natal star or unknown binary companions, (2) heating by gas-grain collision, or (3) gradual thermal annealing when a dust transverse a warm dust shell. We imply gradual annealing may be the most promising. However, it is also accompanied with serious question whether the observable amount of aliphatic $\mathrm{C}-\mathrm{H}$ material, which is easily affected by thermal annealing, can survive through the inner warmer region of a dust shell. The hypothesis is highly speculative and needs to be confirmed by the extensive laboratory works which more precisely express the physical environment around PPNe as well as observations on thermal and dynamical structure of each PPN.

\section{Summary}

We produce QCC films with a hydrocarbon plasma deposition technique. We have obtained emission and absorption spectra of as-deposited and thermally annealed QCC in the $3 \mu \mathrm{m}$ region. The relative strength of $\mathrm{sp}^{2}$ hybridized $\mathrm{CH}$ (aromatic $\mathrm{C}-\mathrm{H}$ ) bonds increases with the annealing temperature with respect to the intensity due to $\mathrm{sp}^{3}$ hybridized $\mathrm{CH}$ (aliphatic $\mathrm{C}-\mathrm{H}$ ) bonds, suggesting graphitization of the QCC films by thermal annealing. Whilst the HRTEM image shows amorphous structure in general (Sakata et al. 1994), carbon onion-like spherules are conspicuous in the QCC annealed at higher temperature. It was consistent with the spectroscopic transformation in the $3 \mu \mathrm{m}$ region, and we think that the graphitization of QCC in the high temperature environment occurs to form carbon onion-like spherules.

We compare the spectral sequence of $3 \mu \mathrm{m}$ spectra of the annealed QCC with observed spectra of PPNe categorized in class B by Geballe (1997) and Tokunaga (1997). Based on the similarity between the laboratory spectra and observed ones particularly in the spectral morphology and the feature to continuum ratio, we propose a scenario 
that carbonaceous dust is formed as condensates similar to QCC samples in the PPN phase, and suffers spectroscopic transformation when driven outward from the star, transversing a warm region of the dust shell. Further observational and laboratory works would confirm this hypothetical view by utilizing high spatial resolution spectroscopy in observations and UV excited QCC emission spectroscopy in laboratory, for instance.

Acknowledgements. We thank the referee, W.W. Duley for his helpful comments that improved the manuscript. We are also grateful to A.T. Tokunaga and T.R. Geballe who kindly provided the spectra of PPNe. MG is supported by the JSPS fellowship.

\section{References}

Bounouh Y., Théye M.L., Dehbi-Alaoui A., Matthews A., Stoquert J.P., 1995, Phys. Rev. B 51, 9597

Buss R.H. Jr., Tielens A.G.G.M., Cohen M., et al., 1993, ApJ 415,250

Cherchneff I., Barker J.R., Tielens A.G.G.M., 1992, ApJ 401, 269

Chiar J.E., Pendleton Y.J., Geballe T.R., Tielens A.G.G.M., 1998, ApJ 507, 281

Dischler B., Bubenzer A., Koidl P., 1983, Solid State Comm. 48, 105

Draine B.T., in: IAU Symp. 135, Interstellar Dust, Allamandola L.J. and Tielens A.G.G.M. (eds.). Kluwer, Dordrecht, p. 313

Duley W.W., 1995, Ap\&SS 224, 217

Frenklach M., Feigelson E.D., 1989, ApJ 380, L43

Geballe T.R., From Stardust to Planetesimals, ASP Conf. Ser. 122, 1997, Pendleton Y.J. and Tielens A.G.G.M. (eds.), p. 119

Geballe T.R., Tielens A.G.G.M., Kwok S., Hrivnak B.J., 1992, ApJ 387, L89

Geballe T.R., van der Veen W.E.C.J., 1990, A\&A 235, L9

Imanishi M., Terada H., Sugiyama K., et al., 1996, PASP 108, 1129
Hrivnak B.J., Guina E.F., Wenxian L., 1995, ApJ 438, 341

Joblin C., Tielens A.G.G.M., Allamandola L.J., Geballe T.R., 1996, ApJ 458, 610

Joblin C., d'Hendecourt L., Léger A., Défourneau D., 1994, A\&A 281, 923

Kwok S., 1993, ARA\&A 31, 63

Mathis J.S., Rumpl W., Nordsieck K.H., 1977, ApJ 217, 425

Meixner M., Skinner C.J., Graham J.R., et al., 1997, ApJ 482, 897

Pendleton Y.J., Sandford S.A., Allamandola L.J., Tielens A.G.G.M., Sellgren K., 1994, ApJ 437, 683

Robertson J., 1991, Prog. Solid St. Chem. 21, 199-333

Sakata A., Wada S., Tababé T., Onaka T., 1984, ApJ 287, L51

Sakata A., Wada S., Onaka T., Tokunaga A.T., 1990, ApJ 353, 543

Sakata A., Wada S., Tokunaga A.T., et al., 1994, 430, 311

Schnaiter M., Henning Th., Mutschke H., 1999, ApJ 519, 687

Scott A.D., Duley W.W., 1996, ApJ 472, L123

Scott A.D., Duley W.W., Jahani H.R., 1997, ApJ 490, L175

Szczerba R., Omont A., Volk K., Cox P., Kwok S., 1997, A\&A 317,859

Tokunaga A.T., Diffuse Infrared Radiation and the IRTS, ASP Conf. Ser. 124, 1997, Okuda H., Matsumoto T. and Rollig T. (eds.), p. 149

Tokunaga A.T., Sellgren K., Smith R.G., Nagata T., Sakata A., Nakada Y., 1991, ApJ 380, 452

Volk K.M., Kwok S., 1989, ApJ 342, 345

\section{List of objects}

"IRAS $05341+0852$ " on page 8

"IRAS $05341+0852$ " on page 9

"the Egg" on page 9

"the Red Rectangle" on page 9

"IRAS $05341+0852$ " on page 10

"AFGL 618 " on page 11

"IRAS $05341+0852$ " on page 15 